\title{
Application of a Modified Cameca ims 1280 Ion Microprobe to Studies of Presolar Grains, Comet Samples, and Other Early Solar-System Materials.
}

\author{
G. R. Huss*, K. Keil*, A. N. Krot*, E. R. D. Scott*, and G. J. Taylor* \\ * Hawaii Institute of Geophysics and Planetology, University of Hawaii at Manoa, 1680 East-West \\ Road, Honolulu, HI 96822, USA
}

An ion microprobe uses a focused beam of high-energy ions to sputter and ionize material from the surface of a sample. The ions are accelerated into a secondary-ion mass spectrometer, which can measure isotopic ratios and elemental abundances. Two commonly used types of secondary-ion mass spectrometer are time-of-flight machines, which separate ions of different mass based on their arrival time at the detector, and magnetic-sector machines, in which a magnet acts as a prism to separate ions as a function of mass. A key advantage of an ion microprobe over other types of mass spectrometers is its ability to measure polished samples that retain their petrographic context.

Cameca produces two basic types of magnetic-sector ion microprobes, the small-geometry ims $3 \mathrm{f}$ 4f-5f-6f-7f series machines and the large-geometry ims 1270-1280 series machines (Fig. 1). Small geometry Cameca ion probes have been used to study early solar-system materials since the mid 1980's, with pioneering work being done at Washington University in St. Louis and at Caltech. The small-geometry machines are very capable and versatile instruments, particularly in their modern incarnations (ims 6f, 7f), but they have fundamental limitations in sensitivity and mass-resolving power (MRP). The large-geometry Cameca ims 1270 [1], and the SHRIMP ion microprobe built by Anutech, were developed to overcome these limitations, particularly as they affect U-Th-Pb dating. These machines began generating data in about 1990. The first ims 1270 instruments to be used to study early solar system materials were installed at the Tokyo Institute of Technology and at UCLA. Another high-sensitivity machine, the Cameca Nanosims, recently has come on-line to study very small samples, including interplanetary-dust particles, and presolar grains [Messenger, this volume].

The Cameca ims 1280 is the newest incarnation of the large-geometry Cameca ion probe. It uses a new generation of electronics to control the primary-ion column and secondary-ion mass spectrometer, has improved magnet control and better counting systems, and has improved computer control of the slits and apertures. These changes have led to major improvements in performance. For example, the new machines can measure oxygen isotopic compositions of mineral grains at a spatial scale of $\sim 25$ microns with a precision and reproducibility of $\sim 0.5$ permil [2].

The Hawaii Institute of Geophysics and Planetology at the University of Hawaii at Manoa, with support from the Keck Foundation and NASA, is putting together a new ion microprobe laboratory dedicated to the study of early solar-system materials, the W. M. Keck Cosmochemistry Laboratory. The heart of the laboratory is a Cameca ims 1280 ion microprobe. We will upgrade this highly capable instrument with additional state-of-the-art technologies to address the unique challenges of research on early solar-system materials. To facilitate the study of tiny grains such as interplanetary dust particles, presolar grains, and comet samples from the STARDUST mission, we will add a secondary-electron detector, precise stage-position control, and a new type of solid-state ion detector called SCAPS $[3,4]$. A secondary-electron detector is a primary navigation tool to locate grains in the Nanosims. The secondary-electron image in the ion microprobe can be directly correlated with the secondary-electron image from an SEM to assist in locating grains of interest for measurement. Precise stage control will permit us to map a sample mount in the SEM and then re-occupy the positions of interesting grains in the ion probe for measurement. The SCAPS detector will permit 
direct ion imaging (in contrast to scanning ion imaging, which is also available on the 1280) of finegrained samples and will permit identification of isotopically or chemically anomalous grains at a spatial resolution of a few tenths of a micron [5,6]. Together with the high sensitivity and MRP of the ims 1280, these modifications will make the UH 1280 a powerful tool for studying tiny grains that is competitive with the Nanosims in most respects.

The UH 1280 will also take advantage of the improved hall-probe system for magnet control and a second nuclear-magnetic-resonance magnet-control system to improve the useful MRP of the secondary-ion mass spectrometer. The ims 1280 was designed with an ultimate MRP of $>40,000$ (M/ $\Delta \mathrm{M}, 10 \%$ definition) [1]. However, the magnet-control system only permitted a useful MPR of $\sim 15,000$. With the new magnet control systems, we hope to improve the useful MRP to $\sim 25,000$, which will permit investigation of new parent-daughter isotope systems for short-lived radionuclides and measurements of trace elements without energy filtering, which should improve sensitivity.

With an ims 1280 ion microprobe modified as described above, and with sustained efforts to develop new measurement techniques and capabilities, we expect the W. M. Keck Cosmochemistry Laboratory at the University of Hawaii to be a world leader in the application of the ion microprobe to studies of early solar-system materials for years to come.

References

[1] C. Conty, SIMS VII (1990) 831.

[2] M. Schuhmacher et al., Presented at the $14^{\text {th }}$ International Conference on Secondary Ion Mass Spectrometery, San Diego, Sept 14-19, 2003. PDF summary available on Cameca website.

[3] K. Nagashima et al., Surf. Interf. Anal. 31 (2001) 131.

[4] H Yurimoto et al., Appl. Surf. Sci. 203-204 (2003) 793.

[5] K. Nagashima et al., Nature 428 (2004) 921.

[6] S. Kobayashi et al., Lun. Plan. Sci. XXXVI (2005) abstr. \# 1931 (CD ROM).

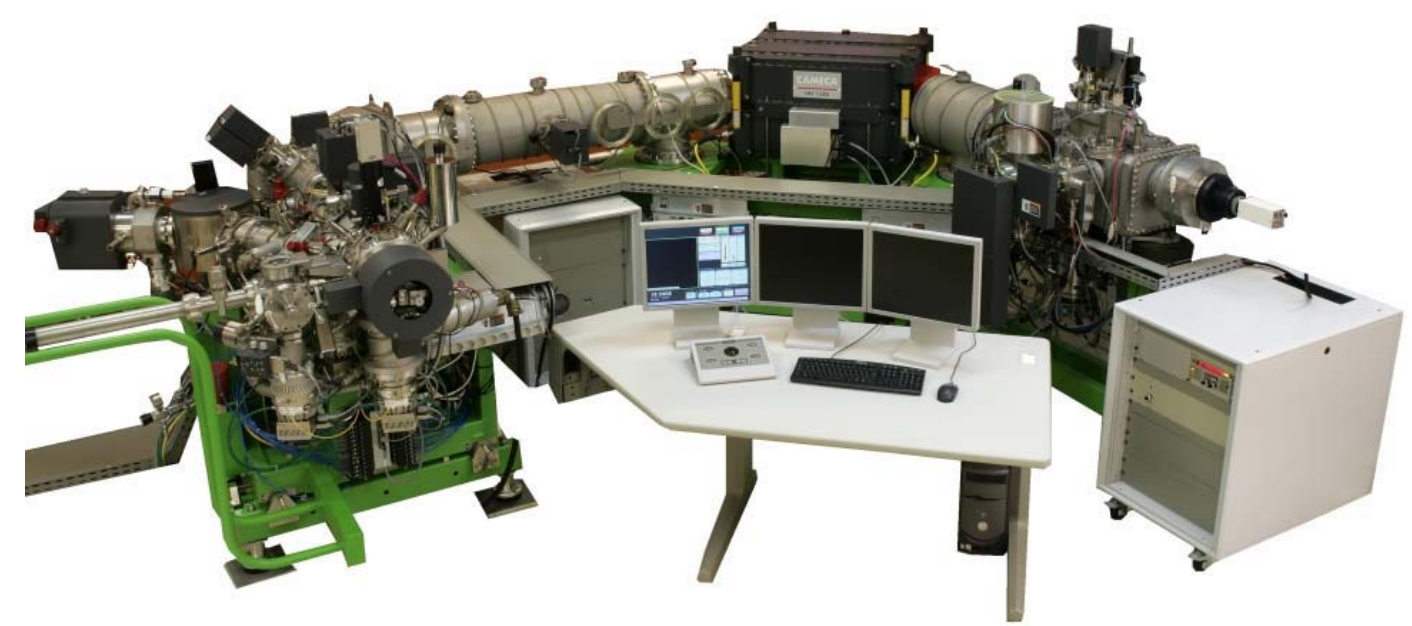

Fig. 1. Cameca ims 1280 ion microprobe. The primary ion column is on the left, the sample sits behind the round gray cover just to the left of the table, and the secondary-ion mass spectrometer extends from the sample in a U-shape to the right. The magnet for the mass spectrometer is in the large gray box above the table. 